\title{
Excision of tricuspid valve with later replacement in endocarditis of drug addiction
}

\section{J S WRIGHT AND J S GLENNIE}

From the Sub-regional Cardio-Thoracic Surgical Centre and the Department of Cardiology, Victoria Hospital, Blackpool, Lancashire, UK

Wright, J S and Glennie, J S (1978). Thorax, 33, 518-519. Excision of tricuspid valve with later replacement in endocarditis of drug addiction. Staphyloccal endocarditis in a drug addict was controlled only after excision of the tricuspid valve. Total absence of the tricuspid valve was tolerated well for 18 months, at which time deteriorating liver function prompted the insertion of a bioprosthetic valve into the tricuspid ring. The haemodynamic and clinical results after two years of follow-up have been excellent.

Infection of the tricuspid valve in drug addicts may require a combined medical and surgical approach. Since a reasonable existence is possible for a limited period without a tricuspid valve, there is a case for a two-stage procedure when intractable infection necessitates valve replacement.

\section{Case report}

A previously healthy man aged 22 presented on 12 October 1973 with a one-month history of febrile illness. He freely admitted to intravenous drug abuse. The features on admission were pyrexia, multiple soft shadows in the chest radiograph (see figure), and a growth of penicillinresistant Staphylococcus aureus in four blood cultures.

Over the next six weeks the signs of gross tri-ळ๊ cuspid regurgitation emerged, and there was@ complete failure of response to intravenous cepha $-\overrightarrow{\vec{\theta}}$ loridine, fusidic acid, and gentamicin. A change of 3 antibiotic regimen (cloxacillin $12 \mathrm{~g}$ and lincomycin? $9 \mathrm{~g}$ daily for eight weeks) brought a temporaryo clinical response, but relapse occurred immediately treatment ceased. The same staphylococcus ap-o peared on blood culture.

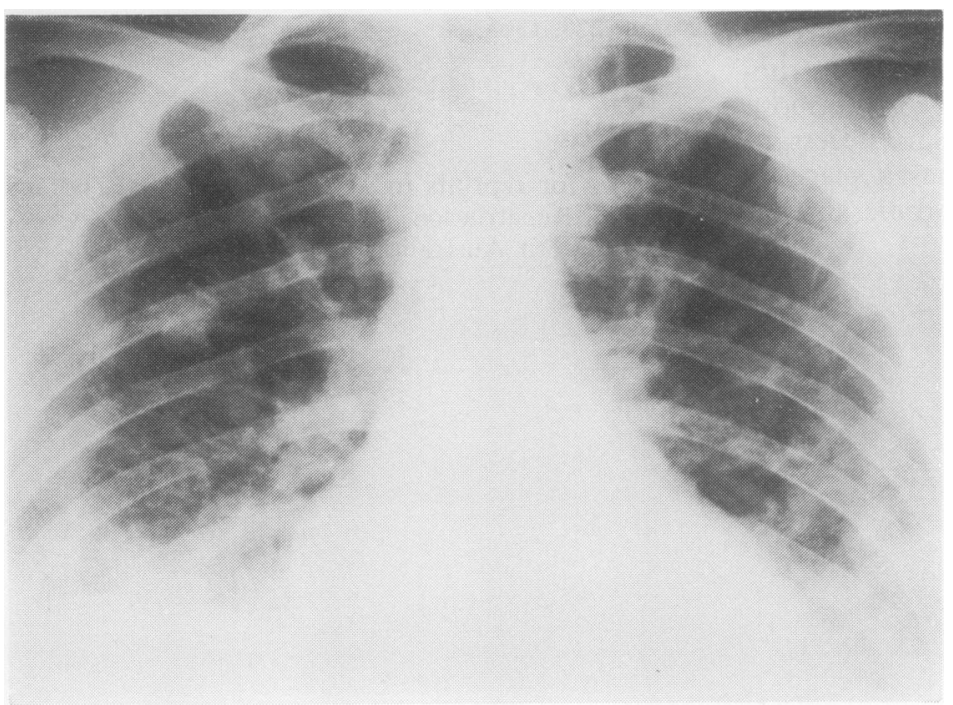

Figure Chest radiograph showing cardiomegaly with some prominence of right atrium and multiple soft shadows in lung fields. 
EXCISION OF TRICUSPID VALVE

Surgery was undertaken on 26 February 1974 using cardiopulmonary bypass. The tricuspid ring was dilated allowing free reflux, and there was one pedunculated vegetation dangling from a tricuspid leaflet. The tricuspid valve leaflets and chordae were excised.

The pyrexia settled the day after surgery and did not recur. Antibiotics were continued for six weeks after surgery, and recovery was uneventful.

\section{PROGRESS IN ABSENCE OF TRICUSPID VALVE}

He was well enough to return to work and to lead an active life over the next 18 months but with excessive fatigue. There was persistent jugular venous engorgement and hepatomegaly with increasing biochemical evidence of hepatic dysfunction.

\section{SECOND OPERATION}

On 9 September 1975 a $35 \mathrm{~mm}$ Hancock porcine heterograft valve was inserted into the tricuspid ring. Recovery was complicated by persistent complete heart block with a nodal escape rate of 60 . Anticoagulants were instituted for three months. Normal atrioventricular conduction returned in six months.

One year after valve replacement the mean right atrial pressure was $7 \mathrm{mmHg}$ with a mean gradient of $3 \mathrm{mmHg}$ across the Hancock valve after exercise (cardiac output $7.46 \mathrm{1} / \mathrm{min}$ ). Right ventriculography showed no significant ventriculoatrial regurgitation.

Two years after his second operation he is leading a normal life. He has no abnormal physical signs and receives no medical treatment. He has married and has been promoted at work. There has been no evidence of return to his addiction.

\section{Discussion}

With the increasing incidence of drug addiction tricuspid endocarditis is becoming more common (Roberts and Buchbinder, 1972). Most studies suggest that the tricuspid valve tends to be singled out for infection in drug addicts (Roberts and
Buchbinder, 1972; Menda and Gorbach, 1973). It is not clear why, and other valves, particularly the aortic, may be affected.

Since the septic emboli are pulmonary, the symptoms and signs are predominantly respiratory and complete the triad of narcotic addiction, pyrexia, and multiple lung lesions, which is so suggestive of tricuspid endocarditis.

Excision of the tricuspid valve in resistant infection was first reported by Arbulu et al (1971), their experience with valve replacement having been uniformly bad. There are two reports of excision with later replacement of the tricuspid valve in addicts (Simberkoff et al, 1974; Arneborn et al, 1977). In these two cases the second operation was carried out at eight and at 12 weeks respectively, and in both cases a metallic prosthesis was used. We think that a longer period should elapse if possible and that a bioprosthesis should be used in the hope that long-term anticoagulant treatment will be less crucial in this group of patients, who are likely to be indifferent attenders.

\section{References}

Arbulu, A, Thoms, N W, Chiscano, A, and Wilson, R F (1971). Total tricuspid valvulectomy without replacement in the treatment of pseudomonas endocarditis. Surgical Forum, 22, 162-164.

Arneborn, P, Björk, V O, Rodriguez, L, and Svanbom, $M$ (1977). Two-stage replacement of tricuspid valve in active endocarditis. British Heart Journal, 39, 1276-1278.

Menda, K B, and Gorbach, S L (1973). Favourable experiences with bacterial endocarditis in heroin addicts. Annals of Internal Medicine, 78, 25-32.

Roberts, W C, and Buchbinder, N A (1972). Rightsided valvular infective endocarditis. American Journal of Medicine, 53, 7-19.

Simberkoff, M S, Isom, W, Smithivas, T, Noriega, E R, and Rahal, J J (1974). Two-stage tricuspid valve replacement for mixed bacterial endocarditis. Archives of Internal Medicine, 133, 212-216.

Requests for reprints to: Mr J S Glennie, Victoria Hospital, Blackpool, Lancs. 